\title{
Therapie der Herzklappenprothesendysfunktion
}

\author{
Therapy of Heart Valve Prosthesis Dysfunction
}

Autoren

Institut
J.-M. Sinning, G. Nickenig

Medizinische Klinik und Poliklinik II, Universitätsklinikum Bonn
Schlüsselwörter

- Klappenprothesendysfunktion

- Thrombose

- Degeneration

- Endokarditis

- TAVI

- Valve-in-Valve

Key words

- valve prosthesis dysfunction

- thrombosis

- degeneration

- endocarditis

- TAVI

valve-in-valve

\section{Bibliografie}

DOI http://dx.doi.org/

10.1055/s-0041-110131

Aktuel Kardiol 2016; 5: 47-51

(c) Georg Thieme Verlag KG

Stuttgart · New York .

ISSN 2193-5203

\section{Korrespondenzadresse}

Priv.-Doz. Dr. med.

Jan-Malte Sinning

Medizinische Klinik und

Poliklinik II

Universitätsklinikum Bonn

Sigmund-Freud-Straße 25

53105 Bonn

Tel.: 0228/2 87-16670

jan-malte.sinning@

ukb.uni-bonn.de

\section{Was ist wichtig?}

- Die europäischen und deutschen Leitlinien für die Behandlung von Patienten mit Herzklappenvitien empfehlen bei Patienten mit Prothesendysfunktion und einem hohen Risiko für eine erneute offene Herzoperation eine individuelle Risiko-Nutzen-Analyse (Alter und Komorbiditäten vs. natürliche Prognose) und in Ermangelung präziser Risikoscores eine individuelle Therapieentscheidung im Herzteam, bestehend aus Kardiologen und Herzchirurgen.

- Bei Verdacht auf Restenosierung einer Herzklappenprothese sollte der Prothesengradient optimalerweise mit den direkt postoperativ erhobenen, individuellen Voruntersuchungen verglichen werden, da dies aussagekräftiger ist als der Vergleich mit theoretischen Grenzwerten für die jeweilige Klappenprothese.

- Katheterinterventionelle Verfahren ermöglichen uns schon heute, dysfunktionale Herzklappenprothesen zu behandeln: Eine paravalvuläre Leckage kann z. B. katheterinterventionell verschlossen werden oder eine transvalvuläre Insuffizienz bzw. Stenosierung im Sinne einer Degeneration bei biologischen Herzklappenprothesen durch eine kathetergestützte Herzklappenimplantation in „Valve-in-Valve“Technik behandelt werden.

- Bei mehr als 200000 chirurgisch implantierten Herzklappen pro Jahr weltweit wird der kathetergestützten Re-Intervention von degenerierten biologischen Klappen mittels „Valve-in-Valve“-Implantation zukünftig eine deutlich wachsende Rolle zukommen. Bevor jedoch die generelle Empfehlung gegeben werden kann, biologische Herzklappenprothesen vor diesem Hintergrund auch bei jüngeren Patienten zu empfehlen, um eine für mechanische Herzklappen notwendige orale Antikoagulation zu vermeiden, müssen noch mehr Langzeitdaten gesammelt werden.

\section{Herzklappenprothesen $\nabla$}

Die Entscheidung zur Herzklappentherapie und zur Methode (operativ, interventionell oder rein medikamentös) beruht heutzutage auf einer individuellen Risiko-Nutzen-Analyse, die bei den gegebenen Charakteristika der Herzklappenerkrankung und den Komorbiditäten die erwartete natürliche Prognose dem angestrebten Therapieergebnis gegenüberstellt. Die Risikostratifizierung sollte überwiegend auf der klinischen Entscheidung des Herzteams beruhen und um Risikoscores wie z. B. den logistischen EuroSCORE ergänzt werden. In die Entscheidungsfindung sollten sowohl die Lebenserwartung und Lebensqualität als auch die individuelle Entscheidung des Patienten nach eingehender Aufklärung einfließen [1,2].

Die Entscheidung, eine Herzklappe zu ersetzen oder zu rekonstruieren, hängt vorwiegend von der Klappenanatomie, der chirurgischen Expertise und dem Zustand des Patienten ab. Ob eine biologische (Vorteil: keine orale Antikoagulation) oder mechanische (Vorteil: längere Haltbarkeit) Klappe eingesetzt wird, hängt neben dem Patientenwillen im Wesentlichen vom Lebensalter ab. Die richtige Prothesenwahl wie auch die nachfolgende Betreuung der Patienten mit einer Herzklappenprothese sind essenziell für die Verringerung von prothesenassoziierten Komplikationen [1-3].

Da mittlerweile im Falle einer degenerativen Dysfunktion der biologischen Klappenprothese der erneute Ersatz mittels kathetergestützter Klappenimplantation (TAVI, transcatheter aortic valve implantation oder TMVI, transcatheter mitral valve implantation) in sog. „Valve-in-Valve“-Technik erfolgen kann und keine offene Reoperation mehr stattfinden muss, ist in den letzten Jahren 
Tab. 1 Ziel-INR für mechanische Klappenprothesen [1,2].

\begin{tabular}{|c|c|c|}
\hline \multirow[t]{2}{*}{ Prothesenthrombogenität ${ }^{1}$} & \multicolumn{2}{|c|}{ patientenassoziierte Risikofaktoren ${ }^{2}$} \\
\hline & kein Risikofaktor & $\geq 1$ Risikofaktor \\
\hline niedrig & 2,5 & 3,0 \\
\hline intermediär & 3,0 & 3,5 \\
\hline hoch & 3,5 & 4,0 \\
\hline
\end{tabular}

1 Prothesenthrombogenität: niedrig = Carbomedics, Medtronic Hall, St-Jude Medical, ON-X; intermediär = übrige Doppelflügelklappen; hoch = Lillehei-Kaster, Omniscience, Starr-Edwards, Björk-Shiley und andere Kippscheiben-Prothesen

2 Patientenassoziierte Risikofaktoren: Mitral- oder Trikuspidalklappenersatz, vorangegangene Thrombembolie, Vorhofflimmern, Mitralklappenstenose jeglicher Ausprägung, linksventrikuläre Ejektionsfraktion $<35 \%$

der Anteil an biologischen Herzklappenprothesen deutlich gestiegen bzw. hat sich die Altersgrenze weiter nach unten verschoben (bisherige Empfehlung in den Leitlinien: biologische Aortenklappe ab 65 Jahren, biologische Mitralklappe ab 70 Jahren) [1]. Außerdem bietet die interventionelle Kardiologie in den letzten Jahren zunehmend auch die Möglichkeit, paravalvuläre Leckagen sowohl biologischer wie auch mechanischer Herzklappenprothesen mittels katheterbasierter Implantation von sog. „Plugs“ zu verschließen und damit eine Reoperation vermeiden zu helfen [4].

Idealerweise wird 6-12 Wochen nach Herzklappenoperation eine komplette Untersuchung des Patienten durchgeführt inklusive transthorakaler Echokardiografie mit Bestimmung des Gradienten über der Prothese, um beim Auftreten von kardialen Symptomen einen Referenzwert für den individuellen Patienten zu haben. Der Prothesengradient hängt nicht nur von der Prothesengröße (Durchmesser) und -position ab, sondern variiert auch je nach Klappentyp und -hersteller [1,2,5].

Die Notwendigkeit der 3-monatigen Antikoagulation nach Aortenklappenersatz mittels Bioprothese wurde in letzter Zeit infrage gestellt; inzwischen wird niedrig dosiertes ASS als Alternative bevorzugt. Trotz der fehlenden Evidenz wird eine Kombination von niedrig dosiertem ASS und einem Thienopyridin unmittelbar nach TAVI sowie nach MitraClip, gefolgt von lebenslänglichem alleinigem ASS oder Thienopyridin, verabreicht. Bei Patienten mit Vorhofflimmern wird eine Kombination aus Vitamin-K-Antagonist und ASS oder Thienopyridin bevorzugt, sollte aber gegen das erhöhte Blutungsrisiko abgewogen werden [1,2]. Inwieweit eine zumindest kurzfristige orale Antikoagulation nach TAVI bzw. biologischem Aortenklappenersatz der Therapie mit ASS und Thienopyridin überlegen sein könnte zur Verhinderung thrombembolischer Komplikationen, wird aktuell in einer großen randomisierten Studie untersucht (GALILEO-Studie, Rivaroxaban nach TAVI). Die Wahl des optimalen INR bei Verwendung einer mechanischen Herzklappenprothese sollte die Risikofaktoren des Patienten und die Thrombogenität der Prothese berücksichtigen, um eine Klappenthrombose im langfristigen Verlauf zu verhindern ( Tab. 1) [1,2]. Die orale Antikoagulation bei mechanischen Herzklappenprothesen wird auch noch in Zukunft eine Domäne von Vitamin-K-Antagonisten wie Marcumar und Warfarin bleiben, nachdem die randomisierte RE-ALIGN-Studie mit Dabigatran vs. Warfarin bei Patienten mit mechanischen Herzklappenprothesen wegen vermehrter Blutungsereignisse und thrombembolischer Ereignisse unter Dabigatran abgebrochen werden musste. Deshalb sollten neue orale Antikoagulanzien bei diesen Patienten nicht eingesetzt werden [6].
Kurzgefasst

Die richtige Prothesenwahl wie auch die nachfolgende Betreuung von Patienten mit einer Herzklappenprothese sind essenziell für die Verringerung von prothesenassoziierten Komplikationen.

\section{Klappenthrombose \\ $\nabla$}

Eine obstruktive Klappenthrombose sollte bei jedem Patienten angenommen werden, der sich mit neuer Luftnot oder einem frischen embolischen Ereignis vorstellt. Durchleuchtung und die Durchführung eines CTs können sehr nützliche zusätzliche Informationen liefern, wenn eine Klappenprothesenthrombose oder ein Pannus vermutet werden. Schwierig kann im Einzelfall die Abgrenzung zur Endokarditis sein, welche bei Vorliegen von Fieber, positiven Blutkulturen oder weiteren Major- bzw. Minorkriterien nach Dukes zunächst angenommen werden sollte.

Die Risiko-Nutzen-Analyse einer Fibrinolyse sollte Patientencharakteristika sowie lokale Gegebenheiten berücksichtigen $[1,2,7]$. Ein dringender oder notfallmäßiger Prothesenersatz wird bei der obstruktiven Klappenthrombose empfohlen, wenn sich die Patienten in einem kritischen Zustand befinden und keine schweren Begleiterkrankungen aufweisen.

Eine Fibrinolyse sollte erwogen werden:

- bei schwer kranken Patienten, die eine Operation höchstwahrscheinlich nicht überleben,

- in Situationen, in denen eine Operation nicht unmittelbar durchführbar ist,

- bei Thrombose einer Trikuspidal- oder Pulmonalklappenprothese.

Wenn eine Fibrinolyse eingeleitet wird, ist bei instabilen Patienten ein kurzes Protokoll zu empfehlen. Entweder kann intravenöse Alteplase (rt-PA) als 10-mg-Bolus und dann 90 mg über 90 Minuten zusammen mit unfraktioniertem Heparin oder Streptokinase $1500000 \mathrm{U}$ über 60 Minuten ohne Heparin angewendet werden. Bei stabilen Patienten können längere Applikationszeiten gewählt werden [1,2].

Das Management von nicht obstruktiven Prothesenthrombosen hängt maßgeblich vom Auftreten thrombembolischer Ereignisse und der Größe des Thrombus ab. Kurzfristige Kontrollen mittels Echokardiografie und/oder Durchleuchtung sind obligatorisch. Bei den meisten Fällen von kleinen Thrombosen (Länge $<10 \mathrm{~mm}$ ) ist die Prognose für die medikamentöse Therapie günstig. Ein gutes Ansprechen mit einer sukzessiven Auflösung des Thrombus vermeidet eine Operation oder Fibrinolyse. Umgekehrt ist die Operation bei großen, nicht obstruktiven Prothesenthromben $(\geq 10 \mathrm{~mm}) \mathrm{zu}$ empfehlen, die durch eine Embolie kompliziert werden und trotz optimaler Antikoagulation persistieren.

Eine gründliche Abklärung jeder thrombembolischen Episode ist essenziell, um geeignete Maßnahmen einzuleiten. Die Prävention zukünftiger thrombembolischer Ereignisse beinhaltet die Behandlung oder Beseitigung behebbarer Risikofaktoren und die Optimierung der Antikoagulationskontrolle, welche z.B. durch die Selbstmessung des Patienten möglich ist. Niedrigdosiertes ASS ( $\leq 100 \mathrm{mg}$ ) sollte bei diesen Patienten dann zusätzlich gegeben werden $[1,2]$. 

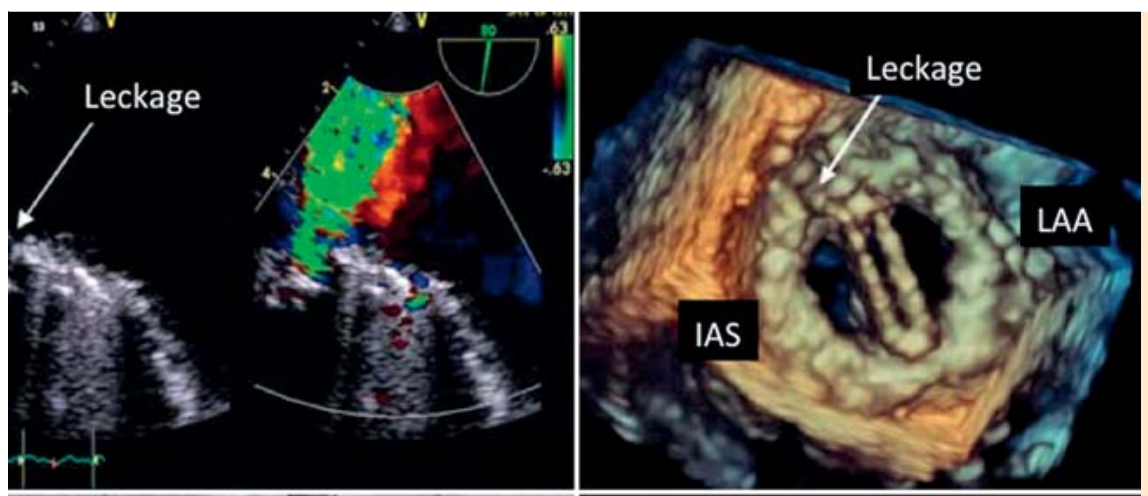

Abb. 1 Perkutaner, 3-D-echokardiografiegesteuerter Verschluss einer paravalvulären Leckage nach mechanischem Mitralklappenersatz.
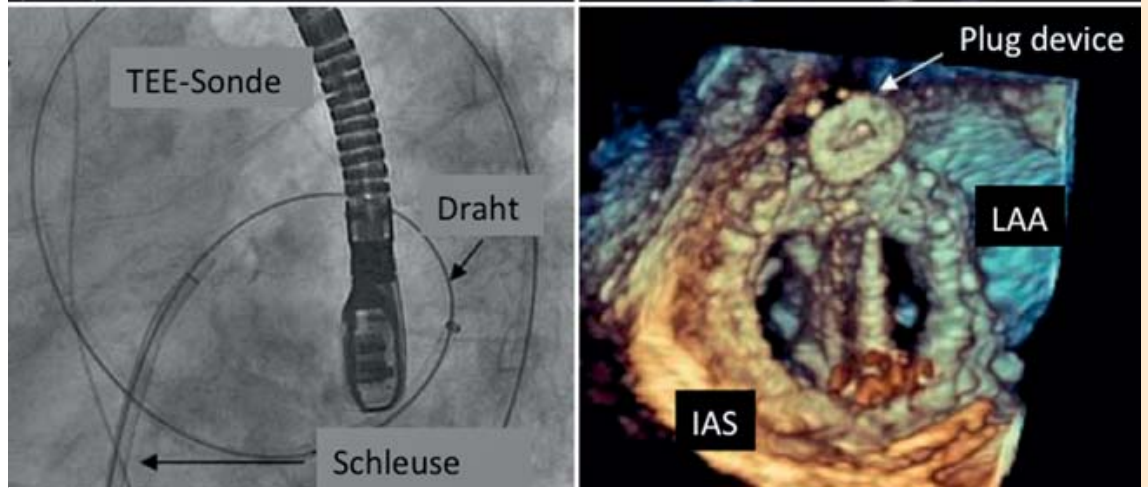

\section{Endokarditis}

$\nabla$

Infektionen von Klappenprothesen machen derzeit etwa 20\% aller Endokarditisfälle aus, mit allerdings steigender Inzidenz. Die Diagnosestellung ist schwieriger als bei Nativklappeninfektionen. Komplizierte Klappenprotheseninfektionen (z.B. mit Abszessoder Fistelbildung), Klappenprotheseninfektionen mit Staphylokokken und die frühe Klappenprothesenendokarditis gehen mit einer schlechten Prognose einher, wenn keine chirurgische Sanierung erfolgt, und sollten vergleichsweise aggressiv therapiert werden. Unkomplizierte Klappenprotheseninfektionen, Klappenprotheseninfektionen, die nicht durch Staphylokokken hervorgerufen werden, und die späte Klappenprothesenendokarditis können oft konservativ behandelt werden (initiale Antibiotikatherapie nach Leitlinie mit Vancomycin, Gentamicin und Rifampicin), erfordern allerdings engmaschige Nachsorgeuntersuchungen. Die Indikation zur transösophagealen Echokardiografie ist bei V.a. eine Herzklappenprothesenendokarditis sehr großzügig zu stellen. Auch nach kathetergestütztem Herzklappenersatz können Endokarditiden auftreten, sodass der jeweilige Echokardiografeur sich mit den jeweiligen Besonderheiten der verwendeten Prothesen auskennen sollte [1,2,7,8].

\section{Paravalvuläre Leckage}

Eine Reoperation wird empfohlen, wenn eine paravalvuläre Leckage (PVL) durch eine Endokarditis hervorgerufen ist oder eine schwere Hämolyse verursacht, die wiederholte Erythrozytentransfusionen nötig macht oder schwere Symptome verursacht. Der kathetergeführte Verschluss der PVL kann bei sorgfältig ausgewählten Patienten erwogen werden, die ein hohes Operationsrisiko besitzen oder bei denen eine Operation kontraindiziert ist, und stellt in erfahrenen Herzzentren mittlerweile eine gute $\mathrm{Al}$ ternative zur Reoperation dar ( Abb. 1) [4,9]. Dabei gilt es, zunächst die PVL zu sondieren und dann über einen eingebrachten Katheter sog. „Plugs“ freizusetzen (z. B. Amplatz vascular plug III oder IV), welche die Leckagen verschließen. Die Plug-Implantation stellt auch eine Therapiemöglichkeit bei Patienten mit mehr als leichtgradiger PVL und damit prognostisch ungünstiger PVL nach TAVI dar, falls eine Nachdilatation nicht zu einer Reduktion der PVL führt [10-12].

\section{Kurzgefasst}

Bevor heute Patienten mit einer paravalvulären Leckage ohne Endokarditisnachweis operiert werden, kann ein Therapieversuch mit einem katheterinterventionellen Verschluss des Leckes versucht werden. 

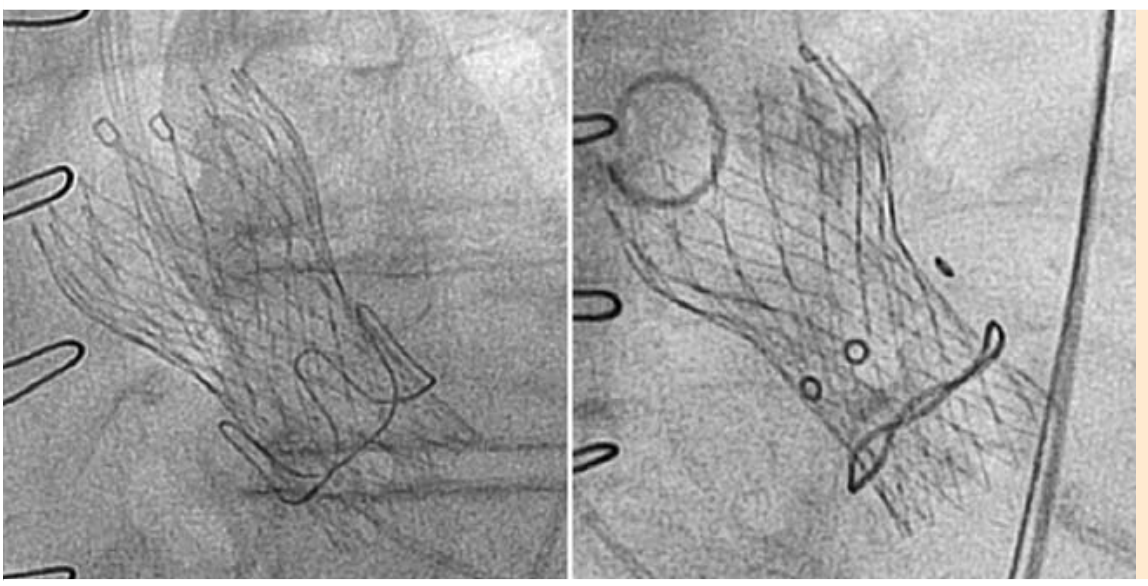

Abb. 2 Beispiele für kathetergestützte Herzklappenimplantation in degenerierte Aortenklappenprothesen.

Tab.2 Echokardiografische Doppler-Parameter zur Evaluation eines erhöhten Gradienten über Aortenklappenprothesen [5].

\begin{tabular}{|c|c|c|c|}
\hline Parameter & normal & $\begin{array}{l}\text { Stenose } \\
\text { wahr- } \\
\text { scheinlich }\end{array}$ & $\begin{array}{l}\text { signifi- } \\
\text { kante } \\
\text { Stenose }\end{array}$ \\
\hline maximale Flussgeschwindigkeit (m/s) & $<3$ & $3-4$ & $>4$ \\
\hline mittlerer Druckgradient $(\mathrm{mmHg})$ & $<20$ & $20-35$ & $>35$ \\
\hline Doppler-Geschwindigkeitsindex ${ }^{1}$ & $\geq 0,30$ & $0,29-0,25$ & $<0,25$ \\
\hline $\begin{array}{l}\text { Akzelerationszeit des aortalen } \\
\text { cw-Doppler-Flussprofils (ms) }\end{array}$ & 80 & $80-100$ & $>100$ \\
\hline
\end{tabular}

1 Verhältnis der maximalen Geschwindigkeit im LVOT (pw-Doppler) zur Aortenklappe (cw-Doppler)

\section{Klappendegeneration}

$\nabla$

Bei Verdacht auf eine Restenosierung einer Herzklappenprothese sollte der Prothesengradient optimalerweise mit den direkt postoperativ erhobenen, individuellen Voruntersuchungen verglichen werden, da dies aussagekräftiger ist als der Vergleich mit theoretischen Grenzwerten für die jeweilige Klappenprothese (O Tab. 2) [5].

Eine Reoperation wird bei symptomatischen Patienten mit einem signifikanten Anstieg des Prothesengradienten oder einer schweren Protheseninsuffizienz empfohlen. Eine Reoperation sollte auch bei asymptomatischen Patienten mit einer signifikanten Prothesendysfunktion erwogen werden, wenn diese ein niedriges Operationsrisiko aufweisen. Perkutane Ballondilatationen sollten bei der Behandlung von stenosierten linkskardialen Klappenprothesen vermieden werden. Eine kathetergeführte „Valvein-Valve“-Implantation kann bei Patienten erwogen werden, die vom Herzteam als inoperabel oder als Hochrisikopatienten eingestuft werden $[1,2,7]$.

Zusätzlich zu den bekannten typischen Dysfunktionsmechanismen chirurgischer Klappenprothesen wie Thrombose, Endokarditis und strukturelle Degeneration durch Insuffizienz und/oder Stenosierung sollten nach kathetergestützter Klappenimplantation mittels TAVI auch noch die folgenden TAVI-spezifischen Dysfunktionsätiologien bei der Nachsorge der Patienten bedacht werden: späte Prothesenembolisation (TAVI-Prothesen werden nicht eingenäht, sondern verankern sich im Kalk der Nativklappe oder z. B. im Gerüst der bereits vorhandenen biologischen Herzklappenprothese mittels Oversizing) oder Prothesendeformation durch äußere Krafteinwirkung wie z.B. nach Reanimation [6,7, 13].

\section{Kurzgefasst}

Neben Thrombose, Endokarditis und struktureller Degeneration als typischen Dysfunktionsmechanismen von Herzklappenprothesen existieren nach der Einführung der kathetergestützten Herzklappenimplantation neben der paravalvulären Leckage 2 weitere Mechanismen für eine Prothesendysfunktion: späte Prothesenembolisation und Prothesendeformation.

\section{Valve-in-Valve-Implantation \\ $\nabla$}

Das internationale VIVID-Registry zeigt, dass kathetergestützte „Valve-in-Valve“-Eingriffe mit einer sehr guten 1-Jahres-Überlebensrate von 83,2\% trotz eines erhöhten Risikos aufgrund zahlreicher Komorbiditäten der behandelten Patienten durchgeführt werden können [14]. Grundsätzlich hatten Patienten mit kleinen Klappenprothesen (häufig sog., ,Patienten-Prothesen-Mismatch“) und restenosierten Klappen eine schlechtere Prognose verglichen mit den anderen in diesem Register eingeschlossenen Patienten. Die Eingriffe sind dabei längst nicht mehr nur im Bereich der Aortenklappen zur Behandlung von dysfunktionalen, biologischen Klappenprothesen möglich ( $\boldsymbol{O A b b . 2}$ ), sondern stellen mittlerweile auch im Bereich der Mitralklappe bei ausgewählten Patienten eine sinnvolle Therapiealternative dar.

\section{Kurzgefasst}

Degenerierte biologische Herzklappenprothesen lassen sich mit einem sehr guten funktionalen Ergebnis und einer guten Prognose, auch bei Hochrisikopatienten, mittels kathetergestützter Herzklappenimplantation in „Valve-in-Valve“-Technik behandeln, um den betroffenen Patienten eine Reoperation zu ersparen.

\section{Ausblick \\ $\nabla$}

Bei mehr als 200000 chirurgisch implantierten Herzklappen pro Jahr weltweit, wird der kathetergestützten Re-Intervention von degenerierten biologischen Klappen mittels „Valve-in-Valve“-Implantation zukünftig eine deutlich wachsende Rolle zukommen. Bevor jedoch die generelle Empfehlung gegeben werden kann, biologische Herzklappenprothesen vor diesem Hintergrund auch bei jüngeren Patienten zu empfehlen, um eine für mechanische 
Herzklappen notwendige orale Antikoagulation zu vermeiden, müssen noch mehr Langzeitdaten gesammelt werden.

Zukünftige Innovationen z.B. in Form von repositionierbaren Herzkatheterklappen der „2. Generation“ werden dazu beitragen, dass Patienten mit einer Dysfunktion ihrer biologischen Herzklappenprothese mit einem guten Ergebnis ohne Reoperation behandelt werden können.

\section{Fazit}

$\nabla$

Die richtige Prothesenwahl wie auch die nachfolgende Betreuung von Patienten mit einer Herzklappenprothese sind essenziell für die Verringerung von prothesenassoziierten Komplikationen. Neben Thrombose, Endokarditis und struktureller Degeneration als typischen Dysfunktionsmechanismen von Herzklappenprothesen existieren nach der Einführung der kathetergestützten Herzklappenimplantation neben der paravalvulären Leckage 2 weitere Mechanismen für eine Prothesendysfunktion: späte Prothesenembolisation und Prothesendeformation. Klappenthrombosen können (gerade bei biologischen Prothesen) nicht selten durch eine (forcierte) orale Antikoagulation behandelt werden. Bei kritisch kranken Patienten kann auch eine Fibrinolyse versucht werden, falls eine Reoperation zu riskant ist. Die Inzidenz der Prothesenendokarditis steigt (ca. 20\% aller Endokarditisfälle). Komplizierte Fälle mit Abszessbildung sollten frühestmöglich chirurgisch therapiert werden. Bevor heute Patienten mit einer paravalvulären Leckage ohne Endokarditisnachweis operiert werden, kann ein Therapieversuch mit einem katheterinterventionellen Verschluss des Leckes versucht werden. Außerdem lassen sich degenerierte biologische Herzklappenprothesen mit einem sehr guten funktionalen Ergebnis und einer guten Prognose, auch bei Hochrisikopatienten, mittels kathetergestützter Herzklappenimplantation in „Valve-in-valve“-Technik behandeln, um den betroffenen Patienten eine Reoperation zu ersparen.

\section{Zusammenfassung \\ $\nabla$}

Die richtige Prothesenwahl wie auch die nachfolgende Betreuung von Patienten mit einer Herzklappenprothese sind essenziell für die Verringerung von prothesenassoziierten Komplikationen. Klappenthrombosen können (gerade bei biologischen Prothesen) nicht selten durch eine (forcierte) orale Antikoagulation behandelt werden. Bei kritisch kranken Patienten kann auch eine Fibrinolyse versucht werden, falls eine Reoperation zu riskant ist. Die Inzidenz der Prothesenendokarditis steigt (ca. 20\% aller Endokarditisfälle). Komplizierte Fälle mit Abszessbildung sollten frühestmöglich chirurgisch therapiert werden. Bevor heute Patienten mit einer paravalvulären Leckage ohne Endokarditisnachweis operiert werden, kann ein Therapieversuch mit einem katheterinterventionellen Verschluss des Leckes versucht werden. Degenerierte biologische Herzklappenprothesen lassen sich mit einem sehr guten funktionalen Ergebnis und einer guten Prognose mittels kathetergestützter Herzklappenimplantation in „Valve-in-Valve“-Technik behandeln, um den betroffenen Patienten eine Reoperation zu ersparen.

\section{Abstract \\ $\nabla$}

The right choice of valve prosthesis as well as the postoperative treatment after valve replacement are the most important aspects to consider, in order to avoid prosthesis-associated complications. Prosthetic valve thrombosis (especially in biological valves) is quite often treated by intensive oral anticoagulation. In critically ill patients, fibrinolysis can also be used if a redo operation is deemed too risky. The incidence of prosthetic endocarditis is on the increase (approximately 20\% of all endocarditis cases). Complicated cases with abscess formation should receive early surgical intervention. Nowadays, attempts can be made to close paravalvular leaks percutaneously in patients without endocarditis, before surgery is performed. Degenerated biological valves can be treated with good results and prognosis with catheterbased valve implantation with the so-called "valve-in-valve" technique, in order to postpone or avoid surgical replacement.

\section{Interessenkonflikt \\ $\nabla$}

PD Dr. Sinning und Prof. Nickenig halten Vorträge für und erhalten Forschungsgelder von Medtronic, Edwards Lifesciences, Abbott und Boston Scientific.

\section{Literatur}

1 Joint Task Force on the Management of Valvular Heart Disease of the European Society of Cardiology (ESC), European Association for CardioThoracic Surgery (EACTS), Vahanian A et al. Guidelines on the management of valvular heart disease (version 2012). Eur Heart J 2012; 33 : 2451-2496

2 Deutsche Gesellschaft für Kardiologie - Herz-und Kreislaufforschung e.V. ESC Pocket Guidelines. Herzklappenerkrankung. Grünwald: Börm Bruckmeier; 2014

3 Sinning JM, Nickenig G. Aortenklappenvitien. Dtsch Med Wochenschr 2014; 139: 1583-1586

4 Rihal CS, Sorajja P, Booker JD et al. Principles of percutaneous paravalvular leak closure. JACC Cardiovasc Interv 2012; 5: 121-130

5 Zoghbi WA, Chambers JB, Dumesnil JG et al. Recommendations for evaluation of prosthetic valves with echocardiography and Doppler ultrasound. J Am Soc Echocardiogr 2009; 22: 975-1014

6 Grube E, Sinning JM, Vahanian A. The Year in Cardiology 2013: valvular heart disease (focus on catheter-based interventions). Eur Heart J 2014; 35: 490-495

7 Sinning JM, Grube E. Transcatheter heart valve failure: the sword of Damocles over our heads? Eur Heart J 2015; 36: 1284-1287

8 Habib G, Hoen B, Tornos P et al. Guidelines on the prevention, diagnosis, and treatment of infective endocarditis (new version 2009): the Task Force on the Prevention, Diagnosis, and Treatment of Infective Endocarditis of the European Society of Cardiology (ESC). Eur Heart J 2009; 30: $2369-2413$

9 Hammerstingl C, Lickfett L, Nickenig G. Real-time three-dimensional transoesophageal echocardiography for guidance of interventional closure of paravalvular leakage. Eur Heart J 2009; 30: 915

10 Sinning JM, Hammerstingl C, Vasa-Nicotera M et al. Aortic regurgitation index defines severity of peri-prosthetic regurgitation and predicts outcome in patients after transcatheter aortic valve implantation. J Am Coll Cardiol 2012; 59: 1134-1141

11 Sinning JM, Vasa-Nicotera M, Chin D et al. Evaluation and management of paravalvular aortic regurgitation after transcatheter aortic valve replacement. J Am Coll Cardiol 2013; 62: 11-20

12 Sinning JM, Vasa-Nicotera M, Werner $N$ et al. Interventional closure of paravalvular leakage after transcatheter aortic valve implantation. Eur Heart J 2012; 33: 2498

13 Roell W, Sinning JM, Werner $N$ et al. How to re-shape a transcatheter heart valve. Eur Heart J 2015; 36: 1287

14 Dvir D, Webb JG, Bleiziffer $S$ et al. Transcatheter aortic valve implantation in failed bioprosthetic surgical valves. JAMA 2014; 312: 162-170 